\section{(A) Check for updates}

Cite this: Polym. Chem., 2020, 11 4768

Received 17th April 2020,

Accepted 22nd June 2020

DOI: 10.1039/d0py00558d

rsc.li/polymers

\title{
Pro-organic radical contrast agents ("pro-ORCAs") for real-time MRI of pro-drug activation in biological systems $\uparrow$
}

\author{
Hung V.-T. Nguyen, (D) $t^{a}, b, c, d$ Alexandre Detappe, (D) $t^{b, c, d, e}$ Peter Harvey, (D) ${ }^{f}$ \\ Nolan Gallagher, ${ }^{a}$ Clelia Mathieu, ${ }^{c, d}$ Michael P. Agius, ${ }^{c, d}$ Oksana Zavidij, ${ }^{c, d}$ \\ Wencong Wang, ${ }^{a}$ Yivan Jiang, ${ }^{a}$ Andrzej Rajca, (D) ${ }^{g}$ Alan Jasanoff, ${ }^{f, h, i}$ \\ Irene M. Ghobrial, ${ }^{c, d}$ P. Peter Ghoroghchian ${ }^{\star b, c, d}$ and Jeremiah A. Johnson (D) $* a, b$
}

\begin{abstract}
Nitroxide-based organic-radical contrast agents (ORCAs) are promising as safe next-generation magnetic resonance imaging (MRI) tools. Nevertheless, stimuli-responsive ORCAs that enable MRI monitoring of prodrug activation have not been reported; such systems could open new avenues for prodrug validation and image-guided drug delivery. Here, we introduce a novel "pro-ORCA" concept that addresses this challenge. By covalent conjugation of nitroxides and drug molecules (doxorubicin, DOX) to the same brush-arm star polymer (BASP) through chemically identical cleavable linkers, we demonstrate that proORCA and prodrug activation, i.e., ORCA and DOX release, leads to significant changes in MRI contrast that correlate with cytotoxicity. This approach is shown to be general for a range of commonly used linker cleavage mechanisms (e.g., photolysis and hydrolysis) and release rates. Pro-ORCAs could find applications as research tools or clinically viable "reporter theranostics" for in vitro and in vivo MRI-correlated prodrug activation.
\end{abstract}

\section{Introduction}

Theranostic agents-systems that simultaneously provide therapeutic and diagnostic modalities-are promising tools for personalized medicine. ${ }^{1-4}$ These agents can, in principle, afford molecular insights into disease states and non-invasively report in real-time on the accumulation, release, and

\footnotetext{
${ }^{a}$ Department of Chemistry, Massachusetts Institute of Technology (MIT), 77 Massachusetts Avenue, Cambridge, Massachusetts 02139, USA

${ }^{b}$ David H. Koch Institute for Integrative Cancer Research, MIT, 77 Massachusetts Avenue, Cambridge, Massachusetts 02139, USA.E-mail: jaj2109@mit.edu, ppg@mit.edu

${ }^{c}$ Department of Medical Oncology, Dana-Farber Cancer Institute, 450 Brookline Avenue, Boston, Massachusetts 02215, USA

${ }^{d}$ Harvard Medical School, 25 Shattuck Street, Boston, Massachusetts 02115, USA ${ }^{e}$ Centre Paul Strauss, 3 Rue de la Porte de l'Hopital, 67000 Strasbourg, France

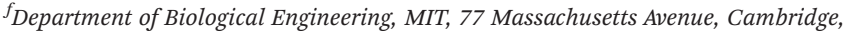
Massachusetts 02139, USA

${ }^{g}$ Department of Chemistry, University of Nebraska, Lincoln, Nebraska 68588, USA ${ }^{h}$ Department of Brain and Cognitive Sciences, MIT, 77 Massachusetts Avenue, Cambridge, Massachusetts 02139, USA

${ }^{i}$ Department of Nuclear Science and Engineering, MIT, 77 Massachusetts Avenue, Cambridge, Massachusetts 02139, USA

$\dagger$ Electronic supplementary information (ESI) available. See DOI: 10.1039/ d0py00558d

$\$$ These authors contributed equally.
}

efficacy of therapeutic agents, thereby enabling real-time therapeutic selection. ${ }^{3,5,6}$ Since their multifunctional requirements are difficult to achieve with small molecules, theranostic systems are often based on nanoscale materials, which themselves possess useful properties such as controllable size and shape, high loading/multiplexing capability, tunable circulation half-life, predictable biodistribution, and targeted tissue accumulation. $^{7-9}$ Indeed, a plethora of theranostic agents based on nanoscale material scaffolds (e.g., liposomes, micelles, dendrimers, and polymers) carrying a wide range of therapeutic entities (e.g., immuno/chemotherapies and gene therapies) and high sensitivity (fluorescence, PET) or high-resolution (CT, MRI) imaging modalities have been reported..$^{10-24}$

"Smart" or "reporter" theranostics that leverage their imaging component to elucidate material-disease tissue interactions such as drug release kinetics, efficacy, or acquired therapeutic resistance are especially powerful. ${ }^{3,25-28}$ Elegant work using fluorescence- or FRET-based reporters of cellular apoptosis or drug release within the tumor microenvironment have been developed. ${ }^{29-36}$ Despite the highly innovative nature of these approaches and their immense utility as research tools, the translational potential of fluorescence imaging may be limited by light penetration depth and tissue autofluorescence. Alternatively, MRI offers high resolution, safety, and 


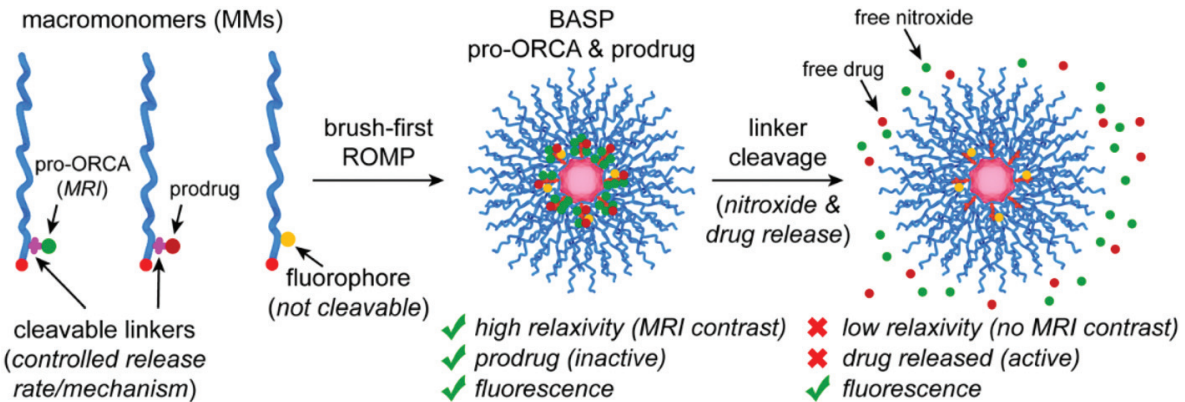

Fig. 1 Design concept for brush-arm star polymer (BASP) pro-ORCAs. Macromonomers (MMs) featuring a pro-ORCA nitroxide (for MRI) and a prodrug (therapeutic) conjugated through cleavable linkers are copolymerized with a non-cleavable fluorophore-conjugated MM via the brush-first ring-opening metathesis polymerization (ROMP) method. The resulting BASP, which carries both the pro-ORCA and prodrug on the same polymer, displays high transverse relaxivity $\left(r_{2}\right)$ enabling $T_{2}$-weighted MRI. Moreover, the prodrug is therapeutically inactive. Upon cleavage of the linkers that connect the nitroxide and drug to the BASP, i.e., pro-ORCA and prodrug activation, the nitroxide relaxivity drops significantly leading to a change of MRI contrast while the drug becomes therapeutically active. Thus, MRI signal changes (in this case, return of negative $T_{2}$ contrast back to baseline) can be correlated with prodrug activation. Meanwhile, the fluoroscence signal should not change significantly before and after nitroxide and drug release, providing a constant imaging handle to visualize the BASP.

established clinical utility. ${ }^{24,25}$ "Reporter" theranostic materials that allow for correlation of drug release with MRI signal changes upon release of Gd-based contrast agents (CAs) or changes to the delivery vehicle itself have been reported. ${ }^{37-40}$ Though metal-based MRI CAs have found widespread clinical utility, they continue to face safety concerns ${ }^{41,42}$ that have led to discontinuation or withdrawal of several products. $^{43-47}$ Metal-free organic radical contrast agents (ORCAs) have been intensely studied in recent years to overcome the safety issues of metal-based CAs; ${ }^{48-58}$ however, to our knowledge, there are no reports of theranostic ORCAs for MRIcorrelated drug release.

We have extensively investigated bottlebrush and related brush-arm star polymer (BASP) polymer architectures for applications in self-assembly, energy storage, drug delivery and imaging, including as ORCAs for MRI. ${ }^{59-71}$ BASPs can be readily covalently conjugated to biocompatible components such as polyethylene glycol (PEG) as well as single or multiple prodrug payloads or imaging agents through the use of branched macromonomers (MMs). ${ }^{59-67,71}$ PEG-based BASPs have displayed excellent safety profiles in mice and higher species, their synthesis is highly scalable and reproducible, and through MM design, the BASP prodrug activation mechanism and rate can be precisely defined ${ }^{59-67}$ BASPs carrying a high concentration of spirocyclohexyl nitroxides $\left(\right.$ chex $\left.^{72}\right)$ currently represent promising ORCAs for MRI, proving useful for $T_{2}$-weighted MRI of tumors in vivo. ${ }^{64-66,71}$

A significant challenge in the field of polymer-based prodrugs, including BASP prodrugs, lies in the real-time monitoring of prodrug activation, i.e., conversion of polymer-bound prodrug into free drug, in biological systems. Prodrug activation kinetics can significantly impact therapeutic outcomes: activation that occurs too quickly or too slowly can be detrimental. ${ }^{62}$ Most prodrugs do not provide facile spectroscopic readouts upon activation; thus, liquid chromatography with tandem mass spectrometry (LC-MS-MS) or inductively coupled plasma mass spectrometry (ICP-MS) are traditionally used to quantify prodrug versus free drug in tissue samples of interest. These methods require tissue harvesting and thus are inherently invasive, ex vivo techniques. The development of noninvasive, safe, "turn-ON/OFF" MRI probes that enables realtime correlation of prodrug activation could offer powerful new research tools and clinical readouts for prodrug development.

Toward addressing this challenge, we describe herein a novel "pro-ORCA" design concept wherein the release of covalently conjugated ORCAs from a BASP scaffold is shown to induce a large ( $\sim 30$-fold) change in magnetic relaxivity that correlates with prodrug activation. Our proof-of-principle proORCA system is based on BASPs that feature both nitroxide ORCAs (for MRI imaging) and the Top2 poison doxorubicin (DOX) covalently conjugated to the same BASP through chemically equivalent linkers that cleave in response to either external or endogenous triggers (Fig. 1). Linker cleavage events, i.e., pro-ORCA and prodrug activation, cause the nitroxide and DOX to diffuse away from the BASP. The former leads to a $\sim 30$ fold change in transverse relaxivity and a concomitant change in MRI contrast, while the latter leads to DOX-induced cell death, enabling MRI-based, real-time monitoring of prodrug activation in a simple, modular format. This basic pro-ORCA concept (Fig. 1) is shown to apply to multiple widely-used triggering mechanisms, variable release kinetics, and in vitro and in vivo models, offering a promising new concept for MRIguided prodrug activation.

\section{Experimental section}

\section{Relaxivity and in vitro measurements by MRI}

Phantom MRI data were acquired in a $12 \mathrm{~cm}$ outer diameter birdcage transceiver for imaging in a $20 \mathrm{~cm}$ bore Bruker 7T Avance III MRI scanner. For relaxivity determination, samples at varying concentrations in PBS buffer were used. For in vitro measurements, samples were prepared as described below, 
and loaded into a 384-well clear polystyrene plate (Thermo Scientific Nunc), which had been pre-cut in half to optimally fit the coil. Unused wells were filled with PBS buffer. $2 \mathrm{~mm}$ slices were imaged through the samples with the field of view of $5 \times 5 \mathrm{~cm}$; and, the data matrices were $256 \times 256$ points. Longitudinal $\left(r_{1}\right)$ and transverse $\left(r_{2}\right)$ relaxivity measurements were acquired using multi-spin multi-echo (MSME) sequences (flip angle $=180^{\circ}$ ). $r_{1}$; TE $=12 \mathrm{~ms}$, TR $=300,350$, $400,450,500,600,800,1000,1200,1500,3000,5000$, $10000 \mathrm{~ms} . r_{2}$; TR $=5000 \mathrm{~ms}, \mathrm{TE}=12,24,36,48,60,72,84$, 96, 108, 120, 132, 144, 156, 168, 280, 192, 204, 216, 228, 240, 252, 264, 276, 288, 300, 312, 324, 336, 348, 360 ms. Custom routines written in Matlab (Mathworks, Natick, MA) were used to reconstruct the images and compute relaxation time constants by fitting image intensity data to exponential decay curves.

For in vitro measurements, cells (A549, MM.1S, and KMS11) were plated at 10000 cells per well in a 96 well plate and incubated overnight. PC2 (17 mg BASP mL ${ }^{-1}, 107 \mu \mathrm{M}$ DOX) was then added, and cells were incubated for another 2 h. Excess PC2 was removed via media wash, and fresh media was added. Cells were then either exposed to UV for $30 \mathrm{~min}(+\mathrm{UV})$ or not $(-\mathrm{UV})$. Cells were then incubated for predetermined time points ( $2 \mathrm{~h}, 6 \mathrm{~h}, 24 \mathrm{~h}$, or $48 \mathrm{~h}$ ), then subject to either MRI or viability assay (CellTiter-Glo, following standard operating procedure). For cells that were imaged by MRI, the media was removed. The wells were then filled with PBS $(50 \mu \mathrm{L})$, mixed with $1 \%$ Triton X-100, transferred to a 384 well plate, and imaged using the MRI protocol described above.

\section{Cell culture}

Human multiple myeloma cells (MM.1S and KMS11, ATCC) were cultured in RPMI media (Thermo Fisher Scientific) and were supplemented with $10 \%$ fetal bovine serum (FBS, VWR), 1\% penicillin/streptomycin (Thermo Fisher Scientific), and 1\% glutamine (Thermo Fisher Scientific). Human lung adenocarcinoma cells (A549, ATCC) were cultured in RPMI media, which was supplemented with $10 \%$ FBS and 1\% penicillin/ streptomycin. Cell lines were authenticated by short tandem repeat DNA profiling and were confirmed to be mycoplasma negative, using the MycoAlert Mycoplasma Testing Kit (Lonza). All cells were housed in $5 \% \mathrm{CO}_{2}$ humidified atmosphere at $37^{\circ} \mathrm{C}$.

\section{In vitro cell viability}

MM.1S, KMS11, and A549 cells were plated at 10000 cells per well overnight in a 96 well plate. The media was then replaced with fresh media containing BASPs at various concentrations. The plate was incubated for $48 \mathrm{~h}$ unless otherwise stated; cell viability was then determined using the CellTiter-Glo assay (Promega).

For viability assays involving DOX-PC or chex-PC, cells were plated at 10000 cells per well in a 96 well plate and incubated overnight. Cells were incubated for $2 \mathrm{~h}$ with DOX-PC or chex-PC at various concentrations. Excess DOX-PC was removed via media wash, and fresh media was added. Cells were then either exposed to UV for $30 \mathrm{~min}$ (+UV) or not (-UV). Viability was evaluated at $48 \mathrm{~h}$ with CellTiter-Glo.

\section{Animal usage}

All animal studies were conducted under federal, state, and local guidelines in accordance with the Public Health Service Policy on Humane Care and Use of Laboratory Animals and the National Institutes of Health Guide for the Care and Use of Laboratory Animals with approval from the Massachusetts Institute of Technology Committee on Animal Care. MR in vivo tumor imaging was performed at the Brain and Cognitive Science Complex at MIT. IVIS imaging was performed at the David H. Koch Institute for Integrative Cancer Research at MIT. NCR/NU mice (female, 8-12 weeks old, Taconic; $n=3$ mice per group) were used for imaging studies.

\section{In vivo therapeutic efficacy of DOX-MHC-BASP}

A549 cells were cultured following the protocol described above to a final concentration of $20 \%$. Cells were then harvested, mixed with Matrigel and sterile $\mathrm{pH}$ 7.4 PBS buffer $(1: 1)$, filtered through sterile $0.2 \mu \mathrm{m}$ filters, and injected subcutaneously $\left(2.0 \times 10^{6}\right.$ cells $)$ into the hind flank of NCR-NU mice. Tumor growth was monitored for 2-4 weeks until appropriate cumulative diameters $(\sim 1 \mathrm{~cm}$, measured by a digital caliper) were achieved. Tumor-bearing mice were then randomized into groups of $n=3$ and injected intratumorally with $50 \mu \mathrm{L}$ of DOX-M at varying concentrations $(1,2.5,5,10 \mathrm{mg}$ BASP $\mathrm{mL}^{-1}$ ). Tumor growth was then accessed via caliper measurements for 25 days.

\section{In vivo MRI instrumentation}

In vivo MRI was acquired using a Bruker 9.4T Biospec MRI scanner using a cross coil volume transmitter and surface receiver configuration. The tumor region was localized in the focal spot of the surface coil, with the animal restrained in order to allow unhindered breathing while minimizing motion around the hind leg and tumor. Axial $T_{1}$ weighted images ( $T_{1}$ WIs) were collected using a RARE pulse sequence with $T_{\mathrm{R}}=$ $721.1 \mathrm{~ms} ; T_{\mathrm{E}(\mathrm{eff})}=11.8 \mathrm{~ms} ;$ RARE factor $=4 ; \mathrm{FOV}=30 \times$ $30 \mathrm{~mm}^{2} ; 256 \times 256$ matrix and 4 averages over 12 slices of $1 \mathrm{~mm}$ thickness and $0 \mathrm{~mm}$ gap, with a total scan time of 2 min $18 \mathrm{~s}$. Axial $T_{2}$ weighted images ( $\left.T_{2} \mathrm{WIs}\right)$ were collected using a RARE pulse sequence with $T_{\mathrm{R}}=4000 \mathrm{~ms} ; T_{\mathrm{E}(\mathrm{eff})}=$ $48 \mathrm{~ms}$; RARE factor $=8$; FOV $=30 \times 30 \mathrm{~mm}^{2} ; 256 \times 256$ matrix and 2 averages over 12 slices of $1 \mathrm{~mm}$ thickness and $0 \mathrm{~mm}$ gap, with a total scan time of 3 min $12 \mathrm{~s}$. Images were analyzed using either ImageJ or custom routines written in Matlab (Mathworks, Natick, MA).

\section{In vivo $\mathrm{MRI}$ in tumor-bearing mice}

Tumor-bearing NCR-NU mice were generated as described above. MRI and NIRF images were acquired for each animal ( $n=3$ mice per group) before injections. BASPs were prepared, passed through a sterile $0.2 \mu \mathrm{m}$ filter, and administered directly into the tumor via intratumoral injections $(50 \mu \mathrm{L}$ of 
$70 \mathrm{mg} \mathrm{mL} \mathrm{m}^{-1}$ BASPs, F, M, or S). Tumor imaging was done at pre-determined time points.

\section{In vivo MRI data analysis}

$T_{2}$-weighted images were inverted before analysis was performed so that high $T_{2}$ regions would appear bright. Signal intensities pre- and post-injection were compared using only slices in which tumors and muscle were clearly visible. Using Image software (v.1.52i), a region of interest (ROI) around each component was manually drawn. The average signal intensities and areas of the ROIs were measured; these data were then normalized against the signal intensity of the muscle tissue. This process was repeated for all relevant slices for a given organ; the sum of these signal intensities was then calculated and divided for the total area, affording the volumeaveraged signal intensity. SNR variations were acquired via normalization against the earliest time point. A detailed step-bystep analysis is included in the ESI. $\dagger$

\section{Ex vivo fluorescence imaging}

For the $24 \mathrm{~h}$ time point, mice were euthanized and their tumors were harvested ( $n=3$ mice per group). Tumor slices were mounted with DAPI staining and imaged with an upright Carl Zeiss microscope with an HXP 120C light source at 20× magnification (DAPI $\lambda_{\mathrm{ex}} / \lambda_{\mathrm{em}}=365 / 445 \mathrm{~nm}$; Cy5.5 $\lambda_{\mathrm{ex}} / \lambda_{\mathrm{em}}=640 /$ $690 \mathrm{~nm} ;$ DOX $\left.\lambda_{\text {ex }} / \lambda_{\text {em }}=470 / 525 \mathrm{~nm}\right)$.

\section{Results and discussion}

We initially targeted photoresponsive linkers to establish the pro-ORCA concept for MRI-correlated prodrug activation. Photocleavable linkers based on ortho-nitrobenzyl (ONB) derivatives have been widely used in the context of protecting group chemistry, drug delivery, and materials applications, ${ }^{73-79}$ and while such linkers may have translational limitations, they offer powerful research tools for triggering specific, light-induced biological responses. DOX was chosen as the therapeutic payloads due to its clinical utility, its established mechanisms of action (in both prodrug and free drug forms), and its inherent fluorescence, the latter of which provides a useful secondary imaging handle for our proof-ofconcept studies. ${ }^{59-61,67,80}$ We have previously shown that conjugation of DOX to BASPs via an ONB linker deactivates the drug (i.e., it is a prodrug), providing no detectable release or cytotoxicity in cell culture over $72 \mathrm{~h}$ in the absence of $365 \mathrm{~nm}$ light. ${ }^{59-61,67}$ Here, we reasoned that this "on/off" nature of light-induced release would simplify our system by enabling a binary readout upon irradiation. A previously reported MM with DOX $^{59}$ and a new MM with chex conjugated via chemically identical ONB linkers (Scheme 1A, see ESI $\uparrow$ for full synthetic details) were prepared; their structures were confirmed by electron paramagnetic resonance spectroscopy (EPR; Fig. $\mathrm{S} 1 \dagger)$, matrix-assisted laser desorption ionization time-offlight mass spectroscopy (MALDI-ToF MS; Fig. S2 $\dagger$ ), and nuclear magnetic resonance (NMR) spectroscopy (Fig. S3†) where appropriate. Four BASPs comprising either 100\% chex (chex-PC) or $100 \%$ DOX (DOX-PC) or different chex:DOX ratios (1:1 and $27: 1$; PC1 and PC2, respectively, Table 1) were synthesized via the brush-first ring-opening metathesis polymerization (ROMP) strategy using an acetal-based bis-norbornene cross-linker (AXL) and Grubbs $3^{\text {rd }}$-generation bis-pyridine complex (G3) (Scheme 1B). ${ }^{59-62,66}$ Leveraging the efficiency of ROMP and the branched MM design, ${ }^{59-66}$ different chex:DOX ratios can be conveniently realized by altering the stoichiometry of MM feed ratios. Gel permeation chromatography (GPC) and dynamic light scattering (DLS) analyses confirmed high MM-to-brush and brush-to-BASP conversions and consistent sizes and size distributions for these four particles; the hydrodynamic diameters $\left(D_{\mathrm{h}}\right)$ were all $\sim 22 \pm$ $5 \mathrm{~nm}$ (Fig. S4a† and Table 1), highlighting the payload-agnostic nature of BASP synthesis. A cy5.5-based MM (Cy-MM) was also incorporated into BASPs that were intended to be deployed for in vivo studies; the cy5.5 dye is non-releasable, providing an independent imaging handle for the BASP scaffold that enables validation of the pro-ORCA design (vide infra). EPR spectroscopy revealed the characteristic broadening associated with conjugation of chex to BASPS (Fig. S4b $\dagger$ ). ${ }^{64-66}$ Moreover, large per-chex $r_{2}$ relaxivity enhancements compared to the model nitroxide 3-CP ( 30-fold), similar to those reported previously upon conjugation of chex to BASPs, ${ }^{64-66}$ were observed (Table 1). This result was encouraging, as it confirmed that chex-conjugated BASPs display high $r_{2}$ values when cleavable pro-ORCAs linkers are used (previous work has exclusively used non-releasable chex linkers) and in the presence of a second payload such as DOX bound to the same polymer.

To examine light-induced pro-ORCA and pro-drug activation, DOX-PC, chex-PC, and PC1 (1:1 chex:DOX) were exposed to $365 \mathrm{~nm}$ light (see ESI $\dagger$ for details). Samples of the reactions were taken at various timepoints and were analyzed by liquid chromatography-MS (LC-MS). The release behaviors were consistent for the three BASPs (Fig. 2A) with maximal release occurring with $\sim 30$ min of light exposure regardless of the payload (i.e., DOX, chex, or 1:1 DOX: chex). As such, an exposure duration of $30 \mathrm{~min}$ was used for subsequent experiments.

To confirm that photoinduced DOX prodrug activation induces cell death, DOX-PC was incubated with suspended multiple myeloma (MM.1S and KMS11) and adherent lung adenocarcinoma (A549) cells for $3 \mathrm{~h}$ to provide sufficient time for uptake. Excess DOX-PC was then removed by media washing, and the cells were exposed to $365 \mathrm{~nm}$ light for $30 \mathrm{~min}$. After incubation for an additional $48 \mathrm{~h}$, cell viability was assessed using the CellTiter-Glo kit (Fig. 2B). Consistent with our expectations, significantly greater toxicity was observed with all cell lines following exposure to $365 \mathrm{~nm}$ light when compared to its absence; note that exposure of cells to $365 \mathrm{~nm}$ light in the absence of DOX-PC induced no observable toxicity (99+\% viability in all 3 cell lines). Moreover, irradiation of cells exposed to chex-PC rather than DOX-PC under the same conditions led to no meaningful effects at similar con- 

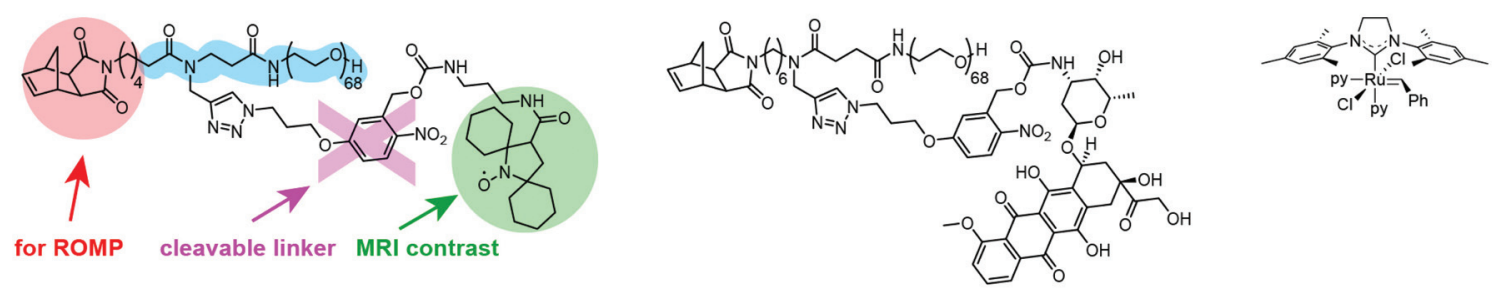

for ROMP cleavable linker MRI contrast

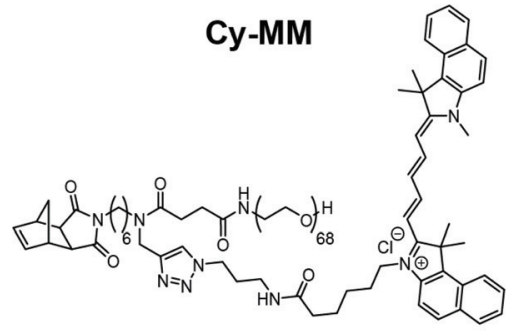

AXL (crosslinker)

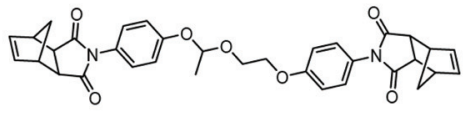

B

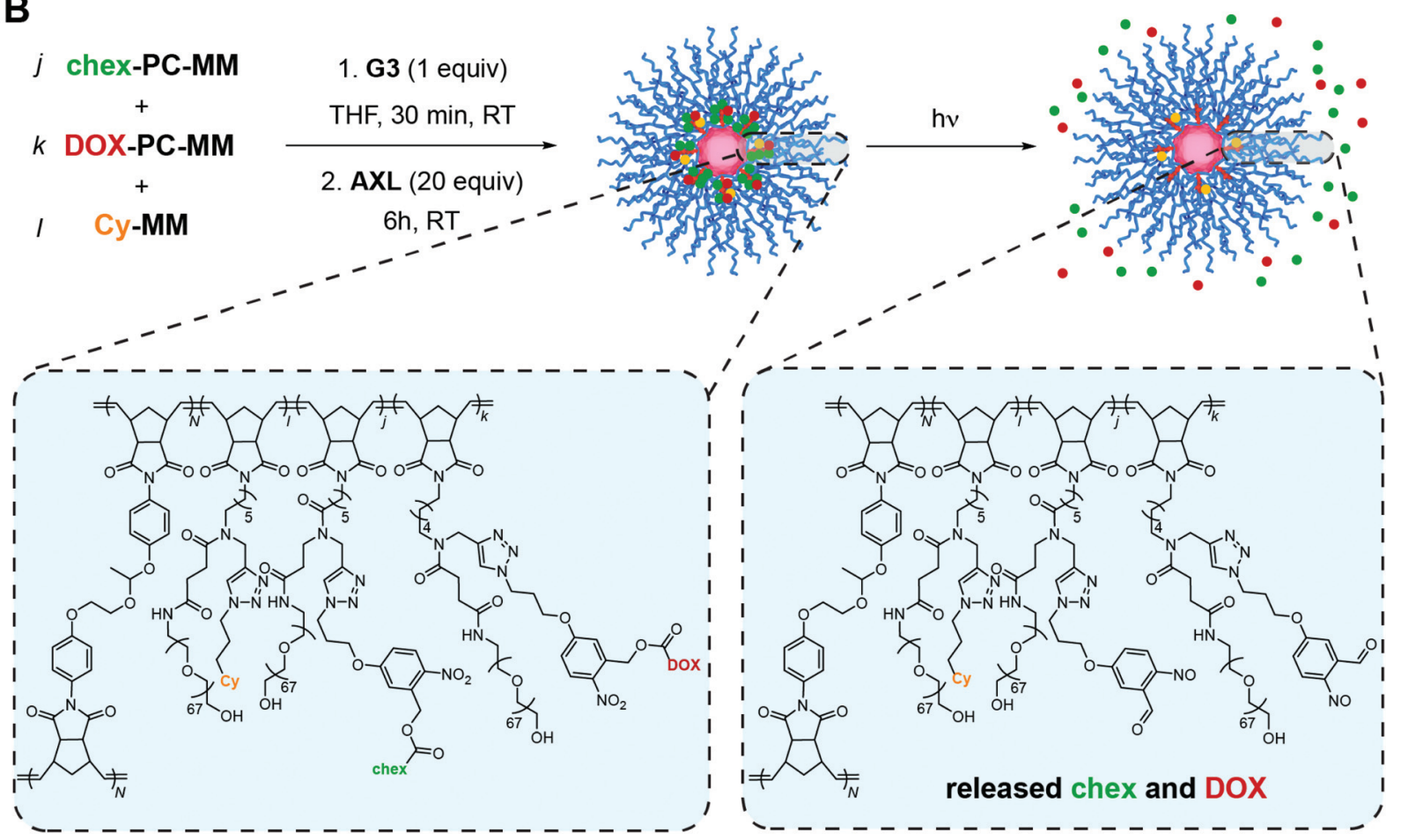

Scheme 1 Photoactivatable BASP pro-ORCA + prodrug design. (A) Chemical structures of MMs chex-PC-MM, DOX-PC-MM, and Cy-MM. The latter two MMs were reported previously, while chex-PC-MM was newly synthesized to enable photocontrolled release of chex. Grubbs $3^{\text {rd }}$ generation bis-pyridine complex (G3, py = pyridine) and acetal-based crosslinker AXL structures are also shown. (B) Brush-first ROMP was conducted by exposing mixtures of the MMs listed above to G3 to generate short bottlebrush polymers. Subsequent addition of AXL induces cross-linking to provide photocleavable BASP "reporter" theranostic agents (e.g., PC1 and PC2). Exposure to light (365 nm) releases chex and DOX from the BASP, inducing concomitant changes in MRI signal and therapeutic efficacy.

centrations (Fig. S5†). These results confirm the low/no in vitro toxicities imparted by the pro-ORCA alone, the polymer components of the BASP, or from $365 \mathrm{~nm}$ light. Only light-triggered DOX prodrug activation induces significant cell death.

Motivated by these results, we examined the use of PC2 for correlation of DOX prodrug activation with changes in MRI contrast in vitro. PC2, which has a chex: DOX ratio of $27: 1$, was designed to maximize the MRI signal at practical concen- trations (i.e., high chex loading) and to still provide sufficient amounts of DOX to induce toxicity following $365 \mathrm{~nm}$ light exposure. Cells were incubated with PC2 (17 mg PC2 mL $\mathrm{mL}^{-1}$; $107 \mu \mathrm{M}$ of conjugated DOX) following the same procedure as described above. The cells were imaged using a 7T MRI scanner (Fig. 2C). MRI SNR was measured as a function of time. In support of our design concept, these SNR values correlated with cell viability (Fig. 2D). Upon irradiation, a dimin- 
Table 1 Characterization data for all BASPs synthesized in this study as well as the model nitroxide 3-CP for comparison

\begin{tabular}{|c|c|c|c|c|c|c|c|}
\hline Sample name & Linker & chex:G3 & DOX:G3 & cy:G3 & $D_{\mathrm{h}} / \mathrm{nm}$ & $r_{1} / \mathrm{mM}^{-1} \mathrm{~s}^{-1}$ & $r_{2} / \mathrm{mM}^{-1} \mathrm{~s}^{-1}$ \\
\hline chex-PC & $\mathrm{PC}$ & 7 & 0 & 0 & $21 \pm 4$ & 0.31 & 5.05 \\
\hline PC2 & PC & 6.75 & 0.25 & 0 & $22 \pm 6$ & 0.29 & 4.91 \\
\hline DOX-S & $\mathrm{HC}, \mathrm{S}$ & 0 & 7 & 0 & $21 \pm 3$ & - & - \\
\hline chex-S & HC, S & 7 & 0 & 0 & $20 \pm 4$ & 0.27 & 5.02 \\
\hline $\mathbf{S}$ & $\mathrm{HC}, \mathrm{S}$ & 6 & 1 & 0.07 & $21 \pm 5$ & 0.29 & 5.01 \\
\hline $\mathbf{M}$ & $\mathrm{HC}, \mathrm{M}$ & 6 & 1 & 0.07 & $20 \pm 5$ & 0.21 & 4.69 \\
\hline DOX-F & $\mathrm{HC}, \mathrm{F}$ & 0 & 7 & 0 & $20 \pm 3$ & - & - \\
\hline chex-F & $\mathrm{HC}, \mathrm{F}$ & 7 & 0 & 0 & $18 \pm 5$ & 0.32 & 4.94 \\
\hline $\mathbf{F}$ & $\mathrm{HC}, \mathrm{F}$ & 6 & 1 & 0.07 & $21 \pm 5$ & 0.26 & 4.85 \\
\hline C & N/A & N/A & N/A & N/A & $20 \pm 4$ & - & - \\
\hline $3-\mathrm{CP}^{51}$ & N/A & N/A & N/A & N/A & N/A & 0.15 & 0.17 \\
\hline
\end{tabular}

$\mathrm{PC}=$ photocleavable; $\mathrm{HC}=$ hydrolytically cleavable (ester-based linker); G3 = Grubbs $3^{\text {rd }}$ generation bis-pyridine complex; $D_{\mathrm{h}}=$ hydrodynamic diameter as measured by dynamic light scattering; per-nitroxide $r_{1}$ and $r_{2}$ values; $\mathrm{S}, \mathrm{M}$, and $\mathrm{F}$ refer to the ester linker used to form the corresponding BASP: slow-, medium-, and fast-releasing.

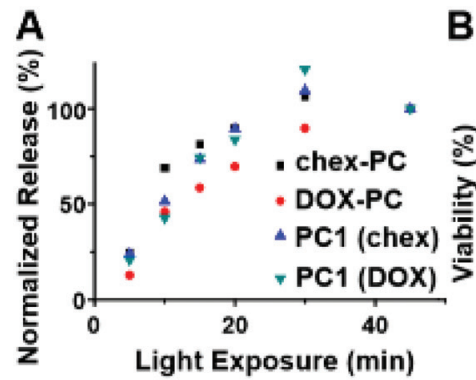

B
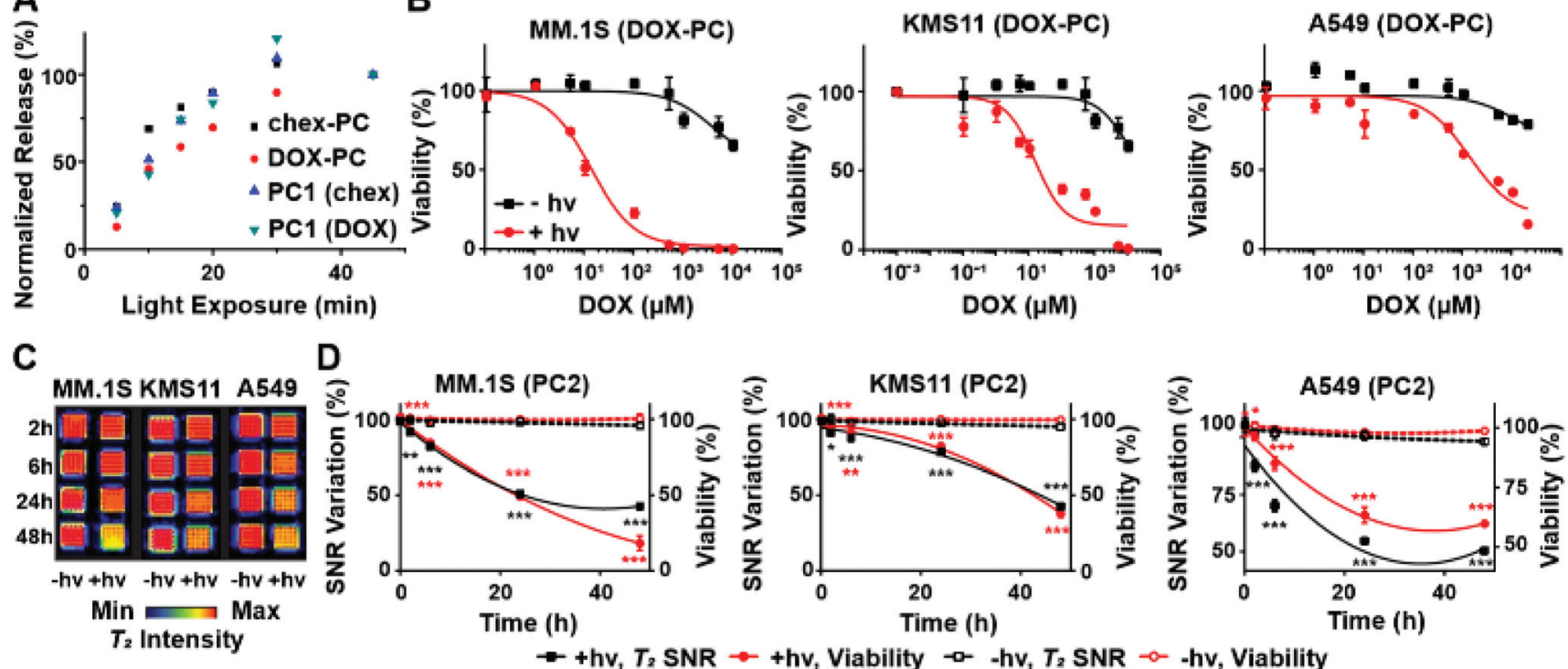

$\rightarrow+h v, T_{2}$ SNR $\rightarrow+h v$, Viability $\rightarrow-$-hv, $T_{2}$ SNR $\rightarrow-h v$, Viability

Fig. 2 In vitro investigations of photocleavable (PC)-BASP pro-ORCAs and prodrugs. (A) Chex and DOX release from PC-BASPs as a function of irradiation $(365 \mathrm{~nm})$ time as determined by liquid chromatography-mass spectrometry (LC-MS). (B) In vitro toxicity of DOX-PC with (red curves) or without (black curves) light-triggered release as examined upon addition to different cancer cell lines (multiple myeloma - MM.1S and KMS11; lung adenocarcinoma - A549). Data are presented as mean \pm SEM $(n=3)$. (C) $T_{2}$-Weighted MRI of cancer cells $2,6,24$, and $48 \mathrm{~h}$ after addition of PC2. False-color inverse-contrast was applied for visualization. (D) MRI signal quantification, revealing changes in SNR that track with incubation time post-UV. Data are presented as mean $\pm \operatorname{SEM~}(n=3)$; statistical analyses between pre- and post-light exposure $( \pm h v)$ for $T_{2}$ MRI SNR variation (black) and cell viability (red) were performed using 2 -tailed student $t$-test (n.s.: not significant, ${ }^{*} p \leq 0.05,{ }^{* *} p \leq 0.01,{ }^{* * *} p \leq 0.001$ ).

ished $T_{2}$ MRI signal was observed as the molar transverse relaxivity of the nitroxide decreases by $\sim 30$-fold upon release (Table 1, $r_{2}$ of water-soluble chex analogue 3-CP $=0.17 \mathrm{mM}^{-1} \mathrm{~s}^{-1}$ compared to PC2, which has a per-chex $r_{2}$ of $4.91 \mathrm{mM}^{-1} \mathrm{~s}^{-1}$ ). Substantial SNR changes were observed in all three irradiated cell lines (Fig. 2D, black solid curves) when compared to nonirradiated cells (Fig. 2D, black dotted curves). For instance, in the case of MM.1S a $57 \pm 3 \%$ decrease in the $T_{2}$ signal was observed from irradiated cells as compared to $5 \pm 2 \%$ for nonirradiated cells. Finally, the MRI SNR data correlated with cyto- toxicity (Fig. 2D, red solid curves) at all time points up to $48 \mathrm{~h}$, indicating that chex and DOX are cleaved and diffuse away from the BASP at similar rates and that the latter was therapeutically active. Altogether, these results demonstrate proofof-principle for the pro-ORCA concept, showing that lightinduced prodrug activation correlates with MRI signal in vitro (Fig. 2D).

Having established proof-of-concept for our pro-ORCA design, we sought to further demonstrate its generally using more translationally-relevant ester-based prodrugs. ${ }^{81}$ Three 
ester linkers were designed and used to prepare pro-ORCAs and DOX prodrugs (Scheme 2A): one that was previously reported to display excellent in vivo efficacy when conjugated to $\mathrm{DOX}^{60,61}$ (referred to herein as "medium") and two novel linkers with steric and electronic properties tuned to afford relatively "fast" and "slow" hydrolysis kinetics in vitro. These linkers utilize the same release mechanism: hydrolytic cleavage of the aromatic ester (rate-determining step), followed by a 1,6-elimination ${ }^{73}$ to release free DOX. Control over their hydrolysis rate is predictable and tunable using rational structural modifications. Using these 3 linkers, 6 different MMs were synthesized: DOX-S-MM; DOX-M-MM; ${ }^{60}$ DOX-F-MM; chex-S-MM; chex-M-MM; and chex-F-MM where S, M, and F correspond to the linker structure: slow, medium and fast, respectively (Scheme 2A, see ESI $\dagger$ for synthetic details). To validate the structures of these MMs (Fig. S6-18†), EPR spectroscopy (Fig. S6, S9, and S12†), NMR spectroscopy (Fig. S7, S10, S13, S15 and S17†), and MALDI-ToF MS (Fig. S8, S11, S14, $\mathrm{S} 16$, and $\mathrm{S} 18 \dagger$ ) were deployed where appropriate.

To confirm their relative release rates, the MMs were incubated in PBS ( $\mathrm{pH}$ 7.4) buffer; the amounts of released DOX or chex were quantified as a function of time via LC-MS. The
A

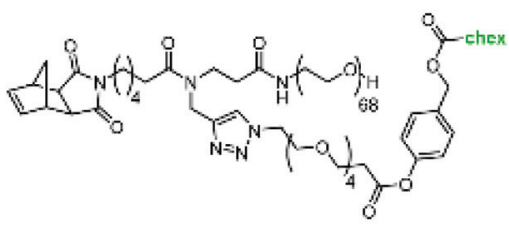

DOX-F-MM

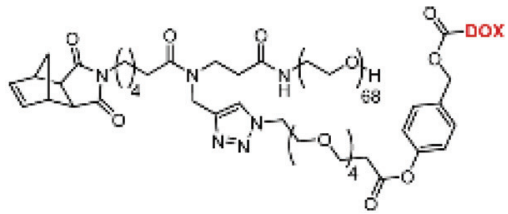

chex-M-MM

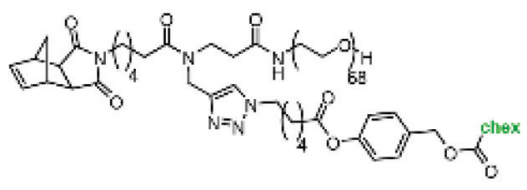

DOX-M-MM

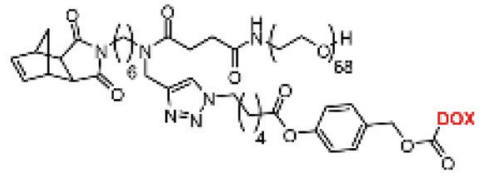

Decreasing Release Rate

$\mathbf{B}$

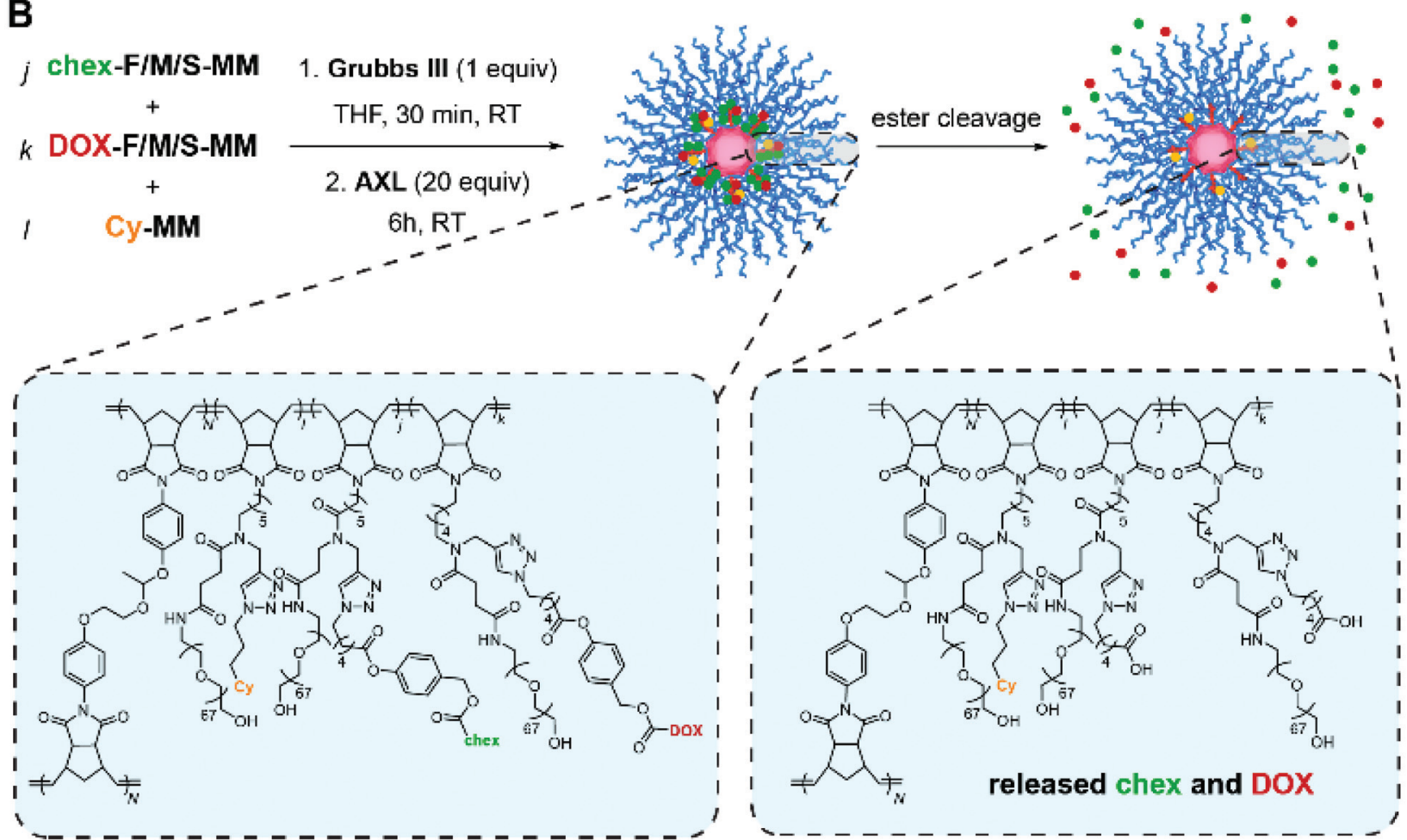

Scheme 2 Hydrolytically labile ester-based BASP pro-ORCAs and prodrugs. (A) Chemical structures of 6 synthesized MMs featuring chex or DOX conjugated via slow, medium, or fast releasing linkers. (B) Brush-first ROMP was used to synthesize BASP "reporter" theranostic agents. Here, release of chex and DOX via ester hydrolysis leads to concomitant MRI and efficacy changes that correlate with the measured hydrolysis rates. 

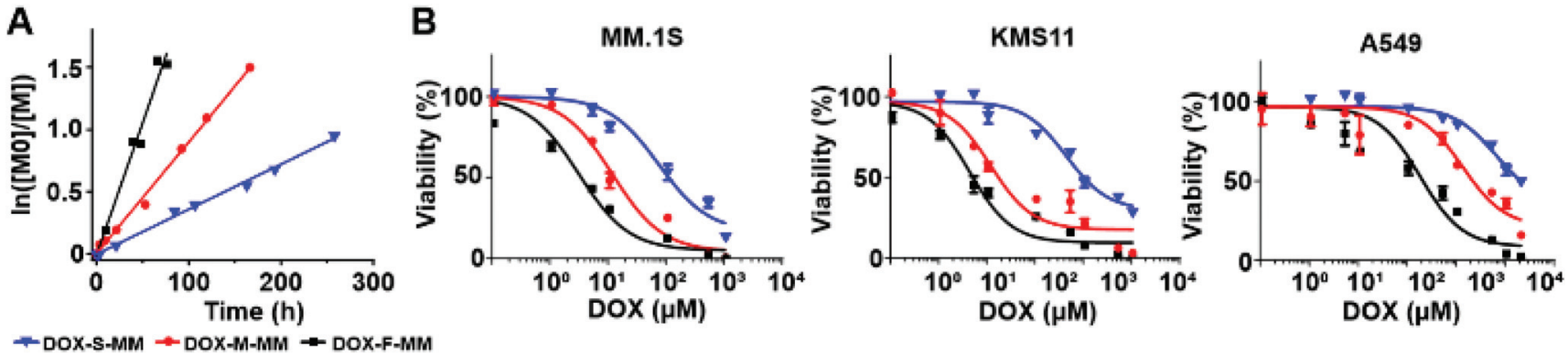

Fig. 3 In vitro investigations of hydrolytically labile MMs and BASPs based on ester linkers. (A) DOX release kinetics from DOX-S-MM (blue), DOX-M-MM (red), and DOX-F-MM (black) in neutral PBS buffer as a function of incubation time as determined by LC-MS. Note: release assays were conducted for MMs rather than BASPs for ease of analysis. Though release is generally much slower from BASPs compared to MMs using the same linker, trends in release rates as a function of linker are preserved. (B) In vitro toxicity of DOX-S (blue), DOX-M (red), and DOX-F (black) in different cell lines. Data are presented as mean $\pm \operatorname{SEM}(n=3)$.

pseudo-first order rate constants for release of DOX from DOX-F-MM, DOX-M-MM, and DOX-S-MM were $21.26 \times 10^{-3}$ $\mathrm{h}^{-1}, 8.95 \times 10^{-3} \mathrm{~h}^{-1}$, and $3.62 \times 10^{-3} \mathrm{~h}^{-1}$, respectively (Fig. 3A and Table S1 $\dagger$ ) while the pseudo-first order rate constants for release of chex from chex-F-MM, chex-M-MM, and chex-S-MM were $8.23 \times 10^{-3} \mathrm{~h}^{-1}, 4.31 \times 10^{-3} \mathrm{~h}^{-1}$, and $1.63 \times 10^{-3} \mathrm{~h}^{-1}$, respectively (Fig. S15 and Table S1 $\dagger$ ). Although these rate constants are not identical across DOX and chex pairs due to differences in the physical properties (e.g., hydrophobicity) of chex and DOX, the DOX : chex release rate ratios were similar: $2.58,2.08$, and 2.22 for the $\mathrm{F}, \mathrm{M}$, and $\mathrm{S}$ pairs, respectively, which enables correlation of DOX prodrug and pro-ORCA activation (vide infra). We note that these in vitro release kinetics studies were conducted using MMs rather than BASPs to ease sample handling and analysis. Though release from BASPs is generally much slower than from MMs using the same linker, trends in release kinetics across different linkers are preserved. ${ }^{62}$ Next, these $6 \mathrm{MMs}$ were used to prepare 6 different singly-loaded BASPs (DOX-F, DOX-M, DOX-S, chex-F, chex-M, chex-S) (Scheme 2B). GPC and DLS results confirmed that the BASP sizes $(\sim 21 \mathrm{~nm})$ were independent of the payload (DOX or chex) or linker (F, M, or S); EPR spectroscopy and MRImeasured per-chex $r_{2}$ values for the three BASP pro-ORCAs showed that the magnetic properties of chex were not meaningfully affected by the linkers (Fig. S20-22 $\dagger$ and Table 1).

Cell viability assays were conducted with the same 3 cell lines used above following $48 \mathrm{~h}$ incubation with these 6 BASPs. As expected, negligible toxicity was observed for the proORCAs lacking DOX (Fig. S23†). In contrast, the toxicity of the DOX prodrug BASPs increased with the rate of DOX release (Fig. 3B); in MM.1S cells, DOX-F, DOX-M, and DOX-S exhibited $\mathrm{IC}_{50}$ values of $3.1 \mu \mathrm{M}, 17.4 \mu \mathrm{M}$, and $113.3 \mu \mathrm{M}$ DOX, respectively. Given that these BASPs have similar sizes and compositions (Table 1) and, thus, expectedly similar cellular internalization rates, this toxicity trend is likely due to varying levels of DOX prodrug activation and release within the $48 \mathrm{~h}$ incubation period. Moreover, the DOX-F viability curve approaches that of free DOX ( $\mathrm{IC}_{50}$ of $2.9 \mu \mathrm{M}$ for MM.1S), suggesting that the majority of DOX may be released from this material in this timeframe (Fig. S24†). Next, viability assessments of MM.1S and A549 were performed as a function of exposure time to each DOX-containing BASP using a fixed dose of $0.5 \mathrm{mg} \mathrm{mL}^{-1}$ BASP (83-86 $\mu \mathrm{M}$ DOX; Fig. S25 $\dagger$ ). Validating our prodrug linker design, the toxicity toward both cell lines followed the same trend: DOX-F $>$ DOX-M $>$ DOX-S.

In order to inform subsequent studies of MRI-guided monitoring of prodrug activation, we studied the in vivo toxicity of DOX-M using a subcutaneous lung adenocarcinoma (A549) model. A549 cells were injected into the hind flank of BALB/c mice (4 groups of $n=3$ mice). Once the tumors reached $\sim 1 \mathrm{~cm}$ in diameter, the mice were randomized and each received a single $50 \mu \mathrm{L}$ intratumoral dose (day 0 ) of 0 (blank), 1, 5, or $10 \mathrm{mg} \mathrm{mL} \mathrm{m}^{-1}$ of DOX-M. Intratumoral administration was selected to maximize tumor MRI SNR and preclude variables of tumor accumulation and pharmacokinetics, thereby simplifying imaging studies for this proof-of-concept work (vide infra). It should be noted that intratumoral drug delivery is a clinically-viable strategy for cancer therapy; ${ }^{82-85} \sim 200$ ongoing clinical trials leverage this method of administration. Moreover, intratumoral administration of BASPs has not been demonstrated before. In our study, significant tumor growth inhibition was observed over the $25 \mathrm{~d}$ period of observation following the administration of DOX-M when compared to the control group (Fig. S26†). Moreover, a dose-dependent response was observed; the $10 \mathrm{mg} \mathrm{mL}^{-1}(1.7 \mathrm{mM}$ ) DOX-M dose provided gradual tumor shrinkage that we reasoned could allow for facile comparisons between different ester linkers in a "reporter" theranostic study.

Based on the above results, a new set of 3 pro-ORCA + prodrug BASPs - F, M, and $\mathbf{S}$ - that each contained both DOX and chex attached to the same polymer (DOX: chex ratio of $1: 6$ to maximize MRI sensitivity) conjugated via the respective fast, medium-, and slow-release ester linkers, as well as $1 \mathrm{~mol} \%$ of non-releasable cy5.5, were synthesized and characterized by GPC, DLS, EPR, and in vitro MRI (Table 1, Fig. S20-22†). The same subcutaneous A549 murine model was employed to correlate DOX prodrug activation from $\mathbf{F}, \mathbf{M}$, and $\mathbf{S}$ with MRI SNR changes in vivo. Each mouse was intratumorally administered either a pro-ORCA-based BASP (F, $\mathbf{M}$, or $\mathbf{S}$ ) or a non-cleavable chex-containing BASP $^{66}$ as a control $(\mathbf{C})$; note that every animal 
received $50 \mu \mathrm{L}$ of a $70 \mathrm{mg} \mathrm{mL}^{-1}$ solution, which corresponded to $\sim 1.7 \mathrm{mM}$ of conjugated DOX, matching the dose used for DOX-M (vide supra). $T_{2}$-Weighted $\mathrm{MR}$ images of the tumors were taken at pre-determined time points, beginning at $30 \mathrm{~min}$ after BASP injection (Fig. 4A). Inversion of image intensity was performed for subsequent analysis in order to aid visualization. In the inverted $T_{2}$-weighted images, signal decreases upon pro-ORCA activation; the image appears brightest in the areas where the pro-ORCA (F, M, or $\mathbf{S}$ ) concentration is the highest. Importantly, SNR variations that correlated with the release kinetics of chex from the corresponding MMs were observed while no changes were observed for C (Fig. 4B), which confirmed the ability of this approach to monitor proORCA activation kinetics in vivo using MRI. For instance, $24 \mathrm{~h}$ post-injection decreases in inverse $T_{2}$-weighted signal of $80 \pm$ $7 \%, 49 \pm 14 \%$, and $9 \pm 1 \%$ were observed for $\mathbf{F}, \mathbf{M}$, and $\mathbf{S}$, respectively; the constant signal observed for non-cleavable BASP C suggests that these BASPs remain in the tumor environment and that there is an insignificant amount of BASP-nitroxide degradation (e.g., via reduction) throughout the timeframe of this study (Fig. 4B and Fig. S27†). ${ }^{66}$ To support our findings that these MRI SNR changes correlated with DOX release, the tumors of mice that had been administered $\mathbf{F}, \mathbf{M}$, and $\mathbf{S}$ were extracted for ex vivo fluorescence imaging to corre-

\section{A}
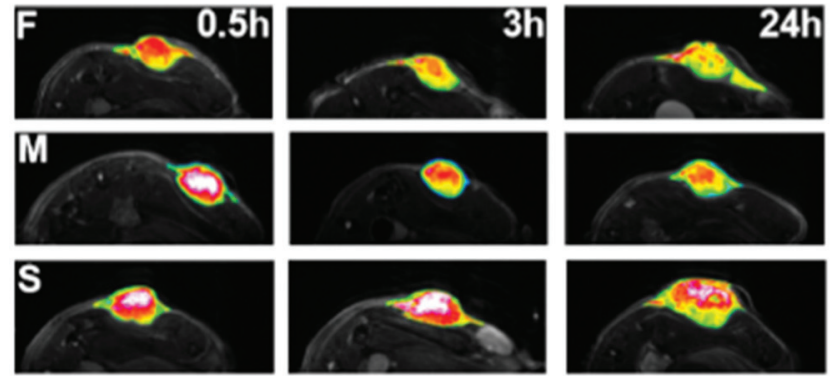

Min

C

DAPI
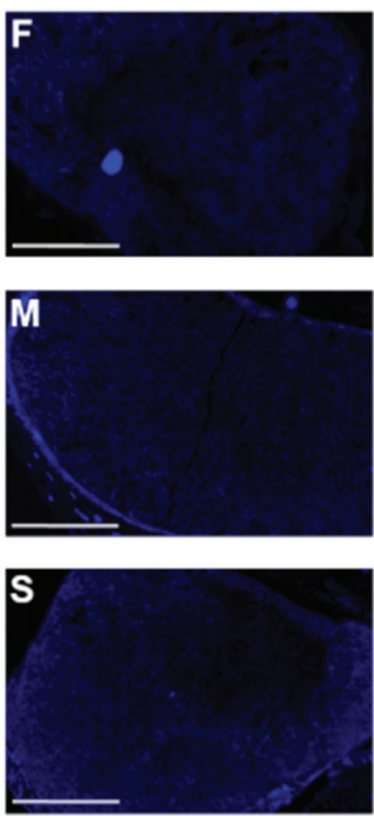

$\operatorname{BASP}(\mathrm{Cy})$
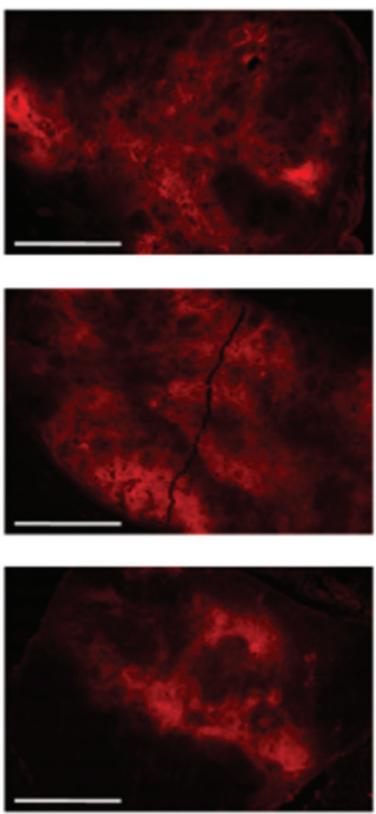

$\operatorname{Max}$

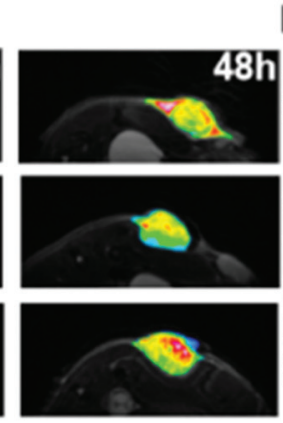

B

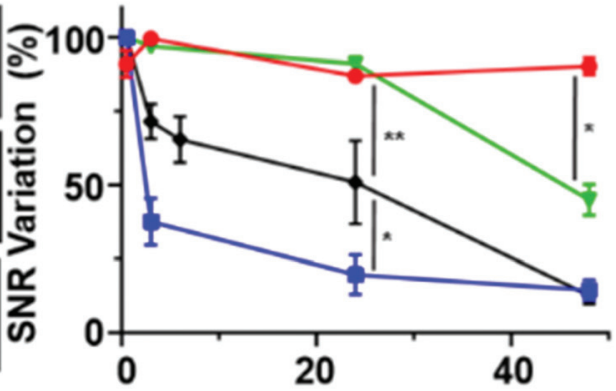

Time Post-Injection (h) $\rightarrow C \rightarrow F \rightarrow M+S$

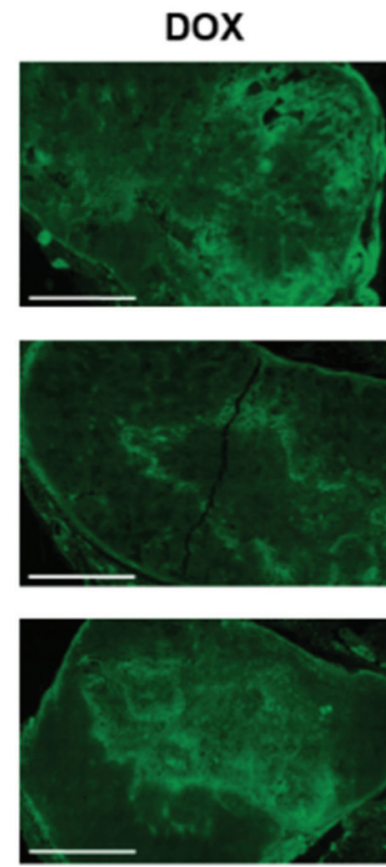

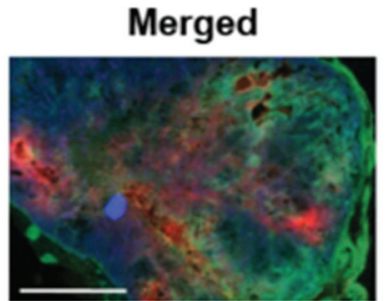
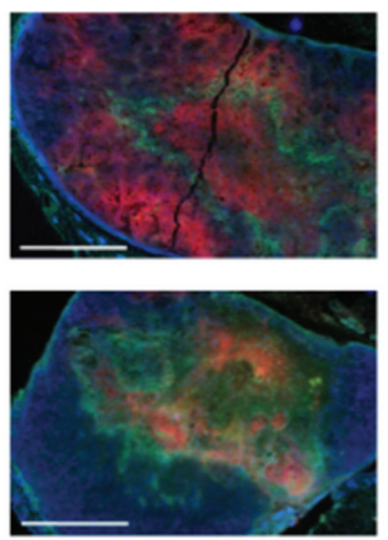

Fig. 4 In vivo examination of hydrolytically labile BASP "reporter" theranostic agents for monitoring drug release in real time. (A) In vivo $T_{2}$-weighted MR images at various time points after intratumoral injection of $\mathrm{F}, \mathrm{M}$, or $\mathrm{S}$ into mice bearing subcutaneous A549 tumors ( $n=3$ mice per group). False-color inverted $T_{2}$-weighted images of the tumor region are overlaid for visualization. These inverted images were used for subsequent analysis. (B) Quantifcation of the changes in contrast over time in mice administered F, M, S, or non-cleavable control BASP (C) $)^{52}$ ( $n=3$ mice per group). Volume-averaged signal intensity across the whole tumor was calculated (see ESI $\dagger$ for full description). Data are presented as the mean \pm SEM; statistical analyses were performed using one-way ANOVA (n.s.: not significant, ${ }^{*} p \leq 0.05,{ }^{* *} p \leq 0.01,{ }^{* * *} p \leq 0.001$ ). (C) Representative fluorescence micrographs of tumors from mice administered F, M, or S 24 h post-injection (scale bar: $2 \mathrm{~mm}$ ). Blue channel: DAPI; red channel: BASP (Cy); green channel: DOX. 
late the intratumoral locations of DOX (via its inherent fluorescence) with those of the BASPs (via cy5.5 fluorescence) (Fig. 4C). We stress that fluorescence is used here as a supporting tool to validate our MRI measurements for this proof-ofconcept study; most drugs are not inherently fluorescent, motivating our development of pro-ORCAs. Gratifyingly, the DOX (green) and BASP (red) signals from the micrographs of tumors treated with the slow-releasing BASP (S) displayed good signal co-localization, suggesting that DOX was still conjugated to the BASP in its prodrug form and in agreement with MRI SNR variations. In contrast, significant separations of DOX (green) and BASP (red) were observed for the fast (F) as well as the medium (M) BASPs, suggesting that DOX release had occurred at sufficiently earlier time points with these constructs. It is, however, difficult to directly compare $\mathbf{F}$ and $\mathbf{M}$, as the fluorescence of DOX is susceptible to alteration by several biological processes. ${ }^{86,87}$ Nonetheless, these results support the fact that $T_{2}$ MRI signal changes induced by pro-ORCA activation correlate with DOX prodrug activation in this system, establishing an example of controlled nitroxide release for correlation of drug release in vivo.

\section{Conclusions}

Herein, we introduced design principles and provided in vitro and in vivo proofs-of-concept for stimuli-responsive pro-ORCAs that enable real-time, non-invasive monitoring of prodrug activation in biological systems using a clinically viable imaging technique (MRI). Specifically, chex ORCAs were conjugated to BASPs through cleavable linkers generating "pro-ORCAs". When attached to the BASP, chex has a large $r_{2}$ value that, when combined with the high density of chex on each BASP, leads to MRI contrast on par with clinically used metal-based contrast agents; cleavage of the linker that connects chex to the BASP leads to a $\sim 30$-fold decrease in $r_{2}$ and concomitant loss of MRI contrast. By combining this pro-ORCA concept with DOX prodrugs conjugated to the same BASP using the same linkers, simultaneous pro-ORCA and prodrug activation occurs, which leads to changes in MRI contrast that correlate with drug release. This concept was demonstrated using 4 different linkers: 1 photocleavable and 3 hydrolytically cleavable linkers with different rates of hydrolysis, in both in vitro and in vivo settings. Regarding the latter, real-time monitoring of drug release via changes in $T_{2}$ MRI signals within a tumor was demonstrated. Altogether, this work establishes a promising platform technology for monitoring prodrug activation in vitro and in vivo. We note that while the subject of this study was the real-time visualization of prodrug activation in biological environments via MRI, the demonstrated linker "plug-andplay" concept can potentially be expanded in the future to reporters of cell apoptosis or immunogenic cell death. Moreover, this approach could potentially be expanded to other therapeutic entities and a range of imaging modalities, providing valuable research tools as well as clinically translatable systems for personalized medicine.

\section{Author contributions}

H. V.-T. N, A. D., P. P. G., I. M. G., and J. A. J conceived the idea. H. V.-T. N, A. D., P. H., N. G., C. M., M. P. A., O. Z., W. W., and Y. J. performed the experiments. H. V.-T. N, A. D., P. P. G., and J. A. J. wrote the manuscript. All authors discussed the results and edited the manuscript.

\section{Conflicts of interest}

There are no conflicts to declare.

\section{Acknowledgements}

We thank the NIH-NCI (1R01CA220468-01 for J. A. J., P. P. G., A. R., A. J.), the NIH (U01-NS090451 for A. J.), and the National Science Foundation (Graduate Research Fellowship for H. V.-T. N.) for support of this research. A. D. acknowledges support from the Fondation Française pour la Recherche contre le Myélome et les Gammapathies, the Philippe Foundation, and the Multiple Myeloma Research Foundation. P. H. acknowledges support from a Wellcome Trust-MIT Postdoctoral Fellowship. P. P. G. acknowledges the generous support of the Charles W. and Jennifer C. Johnson Clinical Investigator Fund as well as the Kathryn Fox Samway Foundation. This work was supported in part by the Koch Institute Support (core) Grant P30-CA14051 from the National Cancer Institute. We thank the Koch Institute Swanson Biotechnology Center for technical support.

\section{Notes and references}

1 M. A. Hamburg and F. S. Collins, N. Engl. J. Med., 2010, 363, 301-304.

2 I. S. Chan and G. S. Ginsburg, Annu. Rev. Genomics Hum. Genet., 2011, 12, 214-244.

3 H. Chen, W. Zhang, G. Zhu, J. Xie and X. Chen, Nat. Rev. Mater., 2017, 2, 17024.

4 R. Kojima, D. Aubel and M. Fussenegger, Curr. Opin. Chem. Biol., 2015, 28, 29-38.

5 X. Chen, S. S. Gambhir and J. Cheon, Acc. Chem. Res., 2011, 44, 841.

6 J. Shi, Z. Xiao, N. Kamaly and O. C. Farokhzad, Acc. Chem. Res., 2011, 44, 1123-1134.

7 S. S. Kelkar and T. M. Reineke, Bioconjugate Chem., 2011, 22, 1879-1903.

8 A. M. Nystrom and K. L. Wooley, Acc. Chem. Res., 2011, 44, 969-978.

9 M. Elsabahy, G. S. Heo, S.-M. Lim, G. Sun and K. L. Wooley, Chem. Rev., 2015, 115, 10967-11011.

10 F. Zhang, S. Zhang, S. F. Pollack, R. Li, A. M. Gonzalez, J. Fan, J. Zou, S. E. Leininger, A. Pavia-Sanders, R. Johnson, L. D. Nelson, J. E. Raymond, M. Elsabahy, D. M. P. Hughes, 
M. W. Lenox, T. P. Gustafson and K. L. Wooley, J. Am. Chem. Soc., 2015, 137, 2056-2066.

11 H. Chen, D. Sulejmanovic, T. Moore, D. C. Colvin, B. Qi, O. T. Mefford, J. C. Gore, F. Alexis, S.-J. Hwu and J. N. Anker, Chem. Mater., 2014, 26, 2105-2112.

12 Y. Li, T.-Y. Lin, Y. Luo, Q. Liu, W. Xiao, W. Guo, D. Lac, H. Zhang, C. Feng, S. Wachsmann-Hogiu, J. H. Walton, S. R. Cherry, D. J. Rowland, D. Kukis, C. Pan and K. S. Lam, Nat. Commun., 2014, 5, 4712.

13 K. Y. Choi, E. J. Jeon, H. Y. Yoon, B. S. Lee, J. H. Na, K. H. Min, S. Y. Kim, S.-J. Myung, S. Lee, X. Chen, I. C. Kwon, K. Choi, S. Y. Jeong, K. Kim and J. H. Park, Biomaterials, 2012, 33, 6186-6193.

14 C. Grange, S. Geninatti-Crich, G. Esposito, D. Alberti, L. Tei, B. Bussolati, S. Aime and G. Camussi, Cancer Res., 2010, 70, 2180-2190.

15 M. Kumar, M. Yigit, G. Dai, A. Moore and Z. Medarova, Cancer Res., 2010, 70, 7553-7561.

16 S.-M. Lee, Y. Song, B. J. Hong, K. W. MacRenaris, D. J. Mastarone, T. V. O'Halloran, T. J. Meade and S. T. Nguyen, Angew. Chem., Int. Ed., 2010, 49, 99609964.

17 S. Kaida, H. Cabral, M. Kumagai, A. Kishimura, Y. Terada, M. Sekino, I. Aoki, N. Nishiyama, T. Tani and K. Kataoka, Cancer Res., 2010, 70, 7031-7041.

18 K. Kim, J. H. Kim, H. Park, Y.-S. Kim, K. Park, H. Nam, S. Lee, J. H. Park, R.-W. Park, I.-S. Kim, K. Choi, S. Y. Kim, K. Park and I. C. Kwon, J. Controlled Release, 2010, 146, 219-227.

19 J. H. Lee, K. Lee, S. H. Moon, Y. Lee, T. G. Park and J. Cheon, Angew. Chem., Int. Ed., 2009, 48, 4174-4179.

20 T. Schluep, J. Hwang, I. J. Hildebrandt, J. Czernin, C. H. J. Choi, C. A. Alabi, B. C. Mack and M. E. Davis, Proc. Natl. Acad. Sci. U. S. A., 2009, 106, 11394-11399.

21 Z. Medarova, W. Pham, C. Farrar, V. Petkova and A. Moore, Nat. Med., 2007, 13, 372-377.

22 N. Nasongkla, E. Bey, J. Ren, H. Ai, C. Khemtong, J. S. Guthi, S.-F. Chin, A. D. Sherry, D. A. Boothman and J. Gao, Nano Lett., 2006, 6, 2427-2430.

23 F. Zhang, Q. Ni, O. Jacobson, S. Cheng, A. Liao, Z. Wang, Z. He, G. Yu, J. Song, Y. Ma, G. Niu, L. Zhang, G. Zhu and X. Chen, Angew. Chem., Int. Ed., 2018, 57, 7066-7070.

24 J. Cheon and J.-H. Lee, Acc. Chem. Res., 2008, 41, 16301640 .

25 T. Lammers, S. Aime, W. E. Hennink, G. Storm and F. Kiessling, Acc. Chem. Res., 2011, 44, 1029-1038.

26 M. H. Lee, J. L. Sessler and J. S. Kim, Acc. Chem. Res., 2015, 48, 2935-2946.

27 A. Sharma, J. F. Arambula, S. Koo, R. Kumar, H. Singh, J. L. Sessler and J. S. Kim, Chem. Soc. Rev., 2019, 48, 771813.

28 M. H. Lee, A. Sharma, M. J. Chang, J. Lee, S. Son, J. L. Sessler, C. Kang and J. S. Kim, Chem. Soc. Rev., 2018, 47, 28-52.

29 A. Kulkarni, P. Rao, S. Natarajan, A. Goldman, V. S. Sabbisetti, Y. Khater, N. Korimerla, V. Chandrasekar,
R. A. Mashelkar and S. Sengupta, Proc. Natl. Acad. Sci. U. S. A., 2016, 113, E2104-E2113.

30 R. Wang, L. Zhou, W. Wang, X. Li and F. Zhang, Nat. Commun., 2017, 8, 14702.

31 S. Bhuniya, S. Maiti, E.-J. Kim, H. Lee, J. L. Sessler, K. S. Hong and J. S. Kim, Angew. Chem., Int. Ed., 2014, 53, 4469-4474.

32 Z. Yang, J. H. Lee, H. M. Jeon, J. H. Han, N. Park, Y. He, H. Lee, K. S. Hong, C. Kang and J. S. Kim, J. Am. Chem. Soc., 2013, 135, 11657-11662.

33 S.-Y. Li, L.-H. Liu, L. Rong, W.-X. Qiu, H.-Z. Jia, B. Li, F. Li and X.-Z. Zhang, Adv. Funct. Mater., 2015, 25, 7317-7326.

34 X. Guo, L. Wang, K. Duval, J. Fan, S. Zhou and Z. Chen, Adv. Mater., 2018, 30, 1705436.

35 X. Li, M. Bottini, L. Zhang, S. Zhang, J. Chen, T. Zhang, L. Liu, N. Rosato, X. Ma, X. Shi, Y. Wu, W. Guo and X.-J. Liang, ACS Nano, 2019, 13, 176-186.

36 M. H. Lee, E.-J. Kim, H. Lee, H. M. Kim, M. J. Chang, S. Y. Park, K. S. Hong, J. S. Kim and J. L. Sessler, J. Am. Chem. Soc., 2016, 138, 16380-163887.

37 C. Kaittanis, T. M. Shaffer, A. Ogirala, S. Santra, J. M. Perez, G. Chiosis, Y. Li, L. Josephson and J. Grimm, Nat. Commun., 2014, 5, 3384.

38 S. Santra, S. D. Jativa, C. Kaittanis, G. Normand, J. Grimm and J. M. Perez, ACS Nano, 2012, 6, 7281-7294.

39 Y. Onuki, I. Jacobs, D. Artemov and Y. Kato, Biomaterials, 2010, 31, 7132-7138.

40 B. L. Viglianti, A. M. Ponce, C. R. Michelich, D. Yu, S. A. Abraham, L. Sanders, P. S. Yarmolenko, T. Schroeder, J. R. MacFall, D. P. Barboriak, O. M. Colvin, M. B. Bally and M. W. Dewhirst, Magn. Reson. Med., 2006, 56, 1011-1018.

41 A. S. Thakor, J. V. Jokerst, P. Ghanouni, J. L. Campbell, E. Mittra and S. S. Gambhir, J. Nucl. Med., 2016, 57, 18331837.

42 V. C. Pierre, M. J. Allen and P. Caravan, J. Biol. Inorg. Chem., 2014, 19, 127-131.

43 Y. X. Wang, Quant. Imaging Med. Surg., 2011, 1, 35-40.

44 Y. X. Wang, World J. Gastroenterol., 2015, 21, 13400-13402.

45 S. Swaminathan, T. D. Horn, D. Pellowski, S. Abul-Ezz, J. A. Bornhorst, S. Viswamitra and S. V. Shah, $N$. Engl. J. Med., 2007, 357, 720-722.

46 I. A. Mendichovszky, S. D. Marks, C. M. Simcock and O. E. Olsen, Pediatr. Radiol., 2008, 38, 489-496.

47 U.S. Food, \& Drug Administration (FDA), 2015; https://www. fda.gov/drugs/drug-safety-and-availability/fda-drug-safetycommunication-fda-strengthens-warnings-and-changesprescribing-instructions-decrease (accessed Dec 16, 2019).

48 F. Hyodo, K. H. Chuang, A. G. Goloshevsky, A. Sulima, G. L. Griffiths, J. B. Mitchell, A. P. Koretsky and M. C. Krishna, J. Cereb. Blood Flow Metab., 2008, 28, 11651174.

49 Z. Zhelev, R. Bakalova, I. Aoki, K.-I. Matsumoto, V. Gadjeva, K. Anzai and I. Kanno, Chem. Commun., 2009, 1, 53-55.

50 N. Bye, O. E. Hutt, T. M. Hinton, D. P. Acharya, L. J. Waddington, B. A. Moffat, D. K. Wright, H. X. Wang, X. Mulet and B. W. Muir, Langmuir, 2014, 30, 8898-8906. 
51 F. Caglieris, L. Melone, F. Canepa, G. Lamura, F. Castiglione, M. Ferro, L. Malpezzi, A. Mele, C. Punta, P. Franchi, M. Lucarini, B. Rossi and F. Trotta, RSC Adv., 2015, 5, 76133-76140.

52 L. Huang, C. Yan, D. Cui, Y. Yan, X. Liu, X. Lu, X. Tan, X. Lu, J. Xu, Y. Xu and R. Liu, Macromol. Biosci., 2015, 15, 788-798.

53 J. M. W. Chan, R. J. Wojtecki, H. Sardon, A. L. Z. Lee, C. E. Smith, A. Shkumatov, S. Gao, H. Kong, Y. Y. Yang and J. L. Hedrick, ACS Macro Lett., 2017, 6, 176-180.

54 S. Garmendia, D. Mantione, S. Alonso-de Castro, C. Jehanno, L. Lezama, J. L. Hedrick, D. Mecerreyes, L. Salassa and H. Sardon, Polym. Chem., 2017, 8, 26932701.

55 M. Dharmarwardana, A. F. Martins, Z. Chen, P. M. Palacios, C. M. Nowak, R. P. Welch, S. Li, M. A. Luzuriaga, L. Bleris and B. S. Pierce, Mol. Pharm., 2018, 15, 2973-2983.

56 J. Wahsner, E. M. Gale, A. Rodriguez-Rodriguez and P. Caravan, Chem. Rev., 2019, 119, 957-1057.

57 K.-A. Hansen and J. P. Blinco, Polym. Chem., 2018, 9, 14791516.

58 O. U. Akakuru, M. Z. Iqbal, M. Saeed, C. Liu, T. Paunesku, G. Woloschak, N. S. Hosmane and A. Wu, Bioconjugate Chem., 2019, 30, 2264-2286.

59 L. Liao, J. Liu, E. C. Dreaden, S. W. Morton, K. E. Shopsowitz, P. T. Hammond and J. A. Johnson, J. Am. Chem. Soc., 2014, 136, 5896-5899.

60 A. X. Gao, L. Liao and J. A. Johnson, ACS Macro Lett., 2014, 3, 854-857.

61 J. C. Barnes, P. M. Bruno, H. V.-T. Nguyen, L. Liao, J. Liu, M. T. Hemann and J. A. Johnson, J. Am. Chem. Soc., 2016, 138, 12494-12501.

62 M. R. Golder, J. Liu, J. N. Andersen, M. V. Shipitsin, F. Vohidov, H. V.-T. Nguyen, D. C. Ehrlich, S. J. Huh, B. Vangamudi, K. D. Economides, A. M. Neenan, J. C. Ackley, J. Baddour, S. Paramasivan, S. W. Brady, E. J. Held, L. A. Reiter, J. K. Saucier-Sawyer, P. W. Kopesky, D. E. Chickering, P. Blume-Jensen and J. A. Johnson, Nat. Biomed. Eng., 2018, 2, 707.

63 H. V.-T. Nguyen, N. M. Gallagher, F. Vohidov, Y. Jiang, K. Kawamoto, H. Zhang, J. V. Park, Z. Huang, M. F. Ottaviani, A. Rajca and J. A. Johnson, ACS Macro Lett., 2018, 7, 472-476.

64 H. V.-T. Nguyen, A. Detappe, N. M. Gallagher, H. Zhang, P. Harvey, C. Yan, C. Mathieu, M. R. Golder, Y. Jiang, M. F. Ottaviani, A. Jasanoff, A. Rajca, I. Ghobrial, P. P. Ghoroghchian and J. A. Johnson, ACS Nano, 2018, 12, 11343-11354.

65 M. A. Sowers, J. R. McCombs, Y. Wang, J. T. Paletta, S. W. Morton, E. C. Dreaden, M. D. Boska, M. F. Ottaviani, P. T. Hammond, A. Rajca and J. A. Johnson, Nat. Commun., 2014, 5, 5460 .
66 H. V.-T. Nguyen, Q. Chen, J. T. Paletta, P. Harvey, Y. Jiang, H. Zhang, M. D. Boska, M. F. Ottaviani, A. Jasanoff, A. Rajca and J. A. Johnson, ACS Cent. Sci., 2017, 3, 800-811.

67 J. A. Johnson, Y. Y. Lu, A. O. Burts, Y. Xia, A. C. Durrell, D. A. Tirrell and R. H. Grubbs, Macromolecules, 2010, 43, 10326-10335.

68 K. Kawamoto, M. Zhong, K. R. Gadelrab, L.-C. Cheng, C. A. Ross, A. Alexander-Katz and J. A. Johnson, J. Am. Chem. Soc., 2016, 138, 11501-11504.

69 Y. Shibuya, H. V.-T. Nguyen and J. A. Johnson, ACS Macro Lett., 2017, 6, 963-968.

70 Y. Shibuya, R. Tatara, Y. Jiang, Y. Shao-Horn and J. A. Johnson, J. Polym. Sci., Part A: Polym. Chem., 2019, 57, 448-455.

71 G. Gil Alvaradejo, H. V.-T. Nguyen, P. Harvey, N. M. Gallagher, D. Le, M. F. Ottaviani, A. Jasanoff, G. Delaittre and J. A. Johnson, ACS Macro Lett., 2019, 8, 473-478.

72 A. Rajca, Y. Wang, M. Boska, J. T. Paletta, A. Olankitwanit, M. A. Swanson, D. G. Mitchell, S. S. Eaton, G. R. Eaton and S. Rajca, J. Am. Chem. Soc., 2012, 134, 15724-15727.

73 J. A. Barltrop, J. Plant and P. Schofield, Chem. Commun., 1966, 22, 822-823.

74 A. Patchornik, B. Amit and R. B. Woodward, J. Am. Chem. Soc., 1970, 92, 6333-6335.

75 J. A. Johnson, M. G. Finn, J. T. Koberstein and N. J. Turro, Macromolecules, 2007, 40, 3589-3598.

76 X. Jiang, C. A. Lavender, J. W. Woodcock and B. Zhao, Macromolecules, 2008, 41, 2632-2643.

77 W. E. Georgianna, H. Lusic, A. L. McIver and A. Deiters, Bioconjugate Chem., 2010, 21, 1404-1407.

78 H. Zhao, E. S. Sterner, E. B. Coughlin and P. Theato, Macromolecules, 2012, 45, 1723-1736.

79 H. Yu, J. Li, D. Wu, Z. Qiu and Y. Zhang, Chem. Soc. Rev., 2010, 39, 464-473.

80 Z. Chen, N. Li, L. Chen, J. Lee and J. J. Gassensmith, Small, 2016, 12, 4563-4571.

81 R. B. Greenwald, A. Pendri, C. D. Conover, H. Zhao, Y. H. Choe, A. Martinez, K. Shum and S. Guan, J. Med. Chem., 1999, 42, 3657-3667.

82 I. Sagiv-Barfi, D. K. Czerwinski, S. Levy, I. S. Alam, A. T. Mayer, S. S. Gambhir and R. Levy, Sci. Transl. Med., 2018, 10, eaan4488.

83 S. Voulgaris, M. Partheni, M. Karamousiz, P. Dimopoulos, N. Papadakis and H. Kalofonos, Am. J. Clin. Oncol., 2002, 25, 60-64.

84 A. Marabelle, L. Tselikas, T. de Baere and R. Houot, Ann. Oncol., 2017, 28, xii33-xii43.

85 M. A. Aznar, N. Tinari, A. J. Rullan, A. R. Sanchez-Paulete, M. E. Rodriguez-Ruiz and I. Melero, J. Immunol., 2017, 198, 31-39.

86 Y. C. Barenholz, J. Controlled Release, 2012, 160, 117-134.

87 P. Mohan and N. Rapoport, Mol. Pharm., 2010, 7, 1959-1973. 